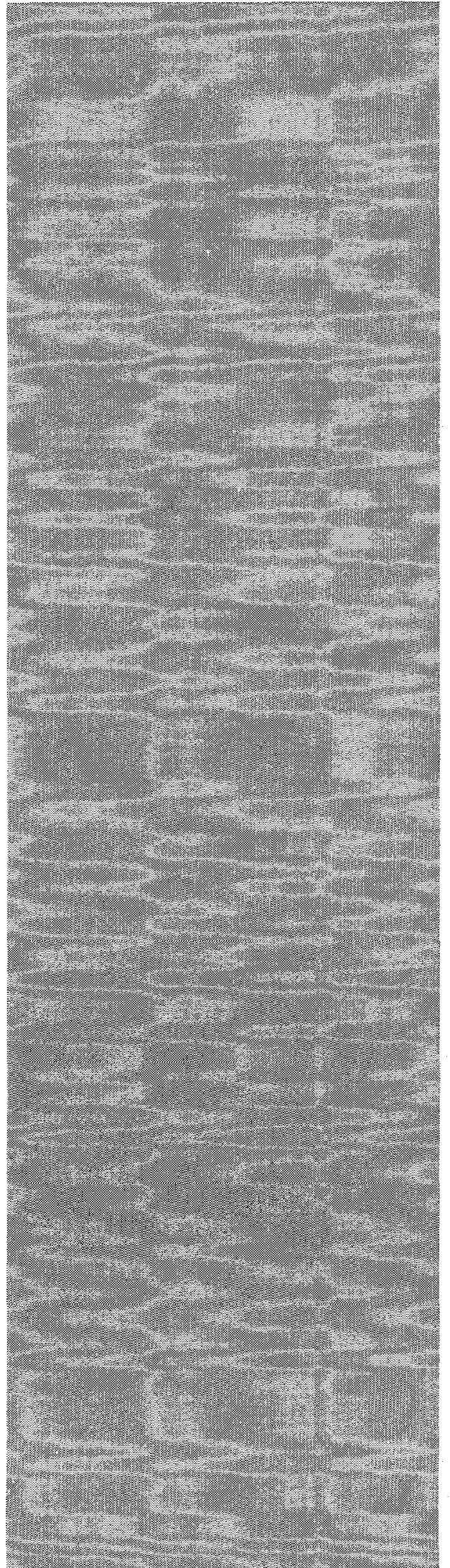

\section{Comparison among Five Hydrodynamic Codes} with a Diverging-Converging Nozzle Experiment

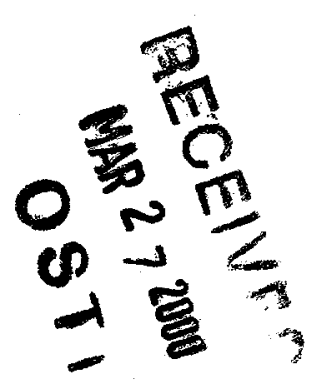

\section{Los Alamos \\ NATIONAL LABORATORY}

Los Alamos National Laboratory is operated by the University of California for the United States Department of Energy under contract W-7405-ENG-36. 
Edited by Patricia W. Mendius, Group CIC-1

An Affirmative Action/Equal Opportunity Employer

This report was prepared as an account of work sponsored by an agency of the United States Government. Neither The Regents of the University of California, the United States

Government nor any agency thereof, nor any of their employees, makes any warranty, express or implied, or assumes any legal liability or responsibility for the accuracy, completeness, or usefulness of any information, apparatus, product, or process disclosed, or represents that its use would not infringe privately owned rights. Reference herein to any specific commercial product, process, or service by trade name, trademark, manufacturer, or otherwise, does not necessarily constitute or imply its endorsement, recommendation, or favoring by The Regents of the University of California, the United States Government, or any agency thereof. The views and opinions of authors expressed herein do not necessarily state or reflect those of The Regents of the University of California, the United States Government, or any agency thereof. Los Alamos National Laboratory strongly supports academic freedom and a researcher's right to publish; as an institution, however, the Laboratory does not endorse the viewpoint of a publication or guarantee its technical correctness. 


\section{DISCLAIMER}

Portions of this document may be illegible in electronic image products. Images are produced from the best available original document. 
Comparison among Five Hydrodynamic Codes with a Diverging-Converging

Nozzle Experiment

L. E. Thode

M. C. Cline

B. G. DeVolder

M. S. Sahota

D. K. Zerkle 


\title{
COMPARISON AMONG FIVE HYDRODYNAMIC CODES WITH A DIVERGING-CONVERGING NOZZLE EXPERIMENT
}

by

L. E. Thode, M. C. Cline, B. G. DeVolder, M. S. Sahota, and D. K. Zerkle

\begin{abstract}
A realistic open-cycle gas-core nuclear rocket simulation model must be capable of a selfconsistent nozzle calculation in conjunction with coupled radiation and neutron transport in three spatial dimensions. As part of the development effort for such a model, five hydrodynamic codes were used to compare with a converging-diverging nozzle experiment. The codes used in the comparison are CHAD, FLUENT, KIVA2, RAMPANT, and VNAP2. Solution accuracy as a function of mesh size is important because, in the near term, a practical three-dimensional simulation model will require rather coarse zoning across the nozzle throat. In the study, four different grids were considered: 1) coarse, radially uniform grid, 2) coarse, radially nonuniform grid, 3) fine, radially uniform grid, and 4) fine, radially nonuniform grid. The study involves code verification, not prediction. In other words, we know the solution we want to match, so we can change methods and/or modify an algorithm to best match this class of problem. In this context, it was necessary to use the higher-order methods in both FLUENT and RAMPANT. In addition, KIVA2 required a modification that allows significantly more accurate solutions for a converging-diverging nozzle. From a predictive point of view, code accuracy with no tuning is an important result. The most accurate codes on a coarse grid, CHAD and VNAP2, did not require any tuning. Our main comparison among the codes was the radial dependence of the Mach number across the nozzle throat. All five codes yielded a very similar solution with fine, radially uniform and radially nonuniform grids. However, the codes yielded significantly different solutions with coarse, radially uniform and radially nonuniform grids. For all the codes, radially nonuniform zoning across the throat significantly increased solution accuracy with a coarse mesh. None of the codes agrees in detail with the weak shock located downstream of the nozzle throat, but all the codes indicated the presence of a weak downstream shock.
\end{abstract}




\section{Introduction}

The concept of an open-cycle gas-core nuclear rocket (OCGCNR) was studied extensively during the $1970 \mathrm{~s}^{1,2}$ Although many small-scale experiments were reported, a self-consistent computational treatment of the engine was not possible. More recently, a computational model for the OCGCNR was reported by Poston-Kammash (PK). ${ }^{3}$ Although the PK model couples hydrodynamics with radiation diffusion and neutron transport, the model assumes an axisymmetric geometry. Moreover, the PK model does not treat the engine nozzle in a self-consistent fashion, which appears to lead to a sensitive dependence of the uranium plasma confinement upon rocket acceleration.

With the advent of large-scale massively parallel computers, a realistic OCGCNR simulation appears possible. Given this capability, a scoping study was performed to determine the requirements for a comprehensive OCGCNR model. ${ }^{4}$ From the scoping study, a realistic OCGCNR model must be capable of a self-consistent nozzle calculation in conjunction with coupled radiation transport and neutron transport in three spatial dimensions. The importance of a self-consistent nozzle calculation leads to the issue of hydrodynamic algorithm accuracy for a converging-diverging nozzle. In particular, solution accuracy as a function of the mesh size is important because a practical OCGCNR calculation will require rather coarse zoning across the nozzle throat.

A converging-diverging nozzle is an important problem with much broader interest than just OCGCNR model development. So, a few validation calculations turned into a modest comparison study of five hydrodynamic codes with an experiment. A converging-diverging nozzle flow offers a rigorous test of computational fluid dynamic methods because of the significant regions of subsonic, transonic, and supersonic flow. In 
addition, the flow in the throat region exhibits large gradients due to the large wall curvature and transonic speed. The experiment selected for this study is the convergingdiverging nozzle investigated by Cuffel et al. ${ }^{5}$ Previously, the experiment was calculated by Prozan (as reported by Cuffel et al. ${ }^{5}$ and Saunders ${ }^{6}$ ), Migdal et al., ${ }^{7}$ Laval, ${ }^{8}$ Serra, ${ }^{9}$ and Cline $^{10}$ using time-dependent methods, and by Prozan and Kooker ${ }^{11}$ using a relaxation procedure. A survey of nozzle methods can be found in Reference 12.

\section{Summary of Hydrodynamic Codes}

The codes CHAD,${ }^{13}$ FLUENT, ${ }^{14}$ KIVA2, ${ }^{15}$ RAMPANT, ${ }^{14}$ and VNAP2 ${ }^{16}$ all differ at a fundamental algorithm level. Moreover, the codes differ significantly in their approach to setup, execution, and solution analysis.

\section{CHAD}

$\mathrm{CHAD}^{13}$ solves the three-dimensional, time-dependent, compressible NavierStokes equations for a turbulent, multimaterial fluid at all flow speeds employing an unstructured mesh on massively parallel computing platforms. The turbulence calculation uses a k- $\varepsilon$ model. CHAD is based on a node-centered, finite-volume method in which all fluid variables are located at computational nodes. CHAD utilizes a variable explicit/implicit upwind method for convection that improves computational efficiency in flows that have large velocity Courant number variations due to velocity or mesh size variations. Strong shocks are handled through the use of a tensor form for the artificial viscosity.

FLUENT

FLUENT is a product of Fluent Incorporated. ${ }^{14}$ The code solves the threedimensional coupled equations for mass, momentum, energy, and chemical species using 
a control volume based finite-difference scheme with the SIMPLE algorithm (SemiImplicit Method for Pressure-Linked Equations). To facilitate calculations on complex geometry, the equations are discretized on a curvilinear grid. We employed the four-level multigrid convergence accelerator option. In this study, a cylindrical coordinate system was used. The code has a large number of options that can be selected by the user for the interpolation and solution methods. Finally, FLUENT offers a steady-state option, which we used to obtain an initial guess for the time-dependent calculations. In all cases, the steady-state and time-dependent solutions were essentially the same.

\section{KIVA2}

The KIVA2 ${ }^{15}$ code solves the three-dimensional, time-dependent, compressible Navier-Stokes equations for the motion of a turbulent, chemically reactive mixture of ideal gases, coupled to the equations for a single-component vaporizing fuel spray. The gas-phase solution procedure is based on the ALE (Arbitrary Lagrangian Eulerian) finitevolume method. The arbitrary structured mesh can conform to curved boundaries. The diffusion terms as well as the terms associated with pressure wave propagation are differenced implicitly using a method similar to the SIMPLE algorithm. The convection terms are differenced using an explicit quasi-second-order upwind scheme that is subcycled.

\section{RAMPANT}

RAMPANT is a product of Fluent Incorporated. ${ }^{14}$ The code solves the coupled three-dimensional equations for mass, momentum, and energy in complex geometry using a finite-volume scheme on an unstructured mesh. We employed the four-level multigrid convergence accelerator option. As in FLUENT, there are a large number of options that 
can be selected by the user for the interpolation and solution methods. Again, as in FLUENT, RAMPANT offers a steady-state option, which we used to obtain an initial guess for the time-dependent calculations. In all cases, the steady-state and timedependent solutions were essentially the same.

VNAP2

The VNAP2 ${ }^{16}$ code solves the two-dimensional, time-dependent, compressible Navier-Stokes equations for the motion of a turbulent, ideal gas. Problems can be solved in either a Cartesian or a cylindrical coordinate system. The grid is nonuniform with body-fitted coordinates in one direction. The unsplit, explicit MacCormack scheme is used to solve the finite-difference equations in the interior of the mesh and a referenceplane characteristic scheme is employed at the boundaries. Artificial viscosity can be used to control algorithm stability.

\section{Code Options}

The study is one of code verification, not prediction. In other words, we know the solution we are attempting to match, so we can change methods and/or modify an algorithm to best match this class of problem. In this context, several different code options were considered for both FLUENT and KIVA2. A summary of the different code options is listed in Table I.

In Table I, CHAD denotes the standard code which is fully implicit with an internal time step calculation. In all cases, the code was run with the Eulerian option.

Three FLUENT options were considered. The Graphics User Interface (GUI) path for the three options is given in Table I. The options reflect differences in the order of the discretization scheme associated with the mass, momentum, and energy equations as well 
as in the interpolation scheme. In all cases, we were able to reduce the residuals by about six orders-of-magnitude in the steady-state mode. So, the differences among the options are not due to different levels of convergence, rather they reflect the differences in the algorithms. In option FLUENT-1, the higher-order schemes are turned off and the "power-law" interpolation scheme is used. In option FLUENT-2, the higher-order schemes are turned on and the "2nd order upwind" option is used. In option FLUENT-3, the higher-order schemes are turned on and the "quick" (quadratic interpolation) option is used.

We considered four options for KIVA2. In Table I, the option KIVA2-1 denotes the standard code using the internally calculated time step. This time step results in at least fifteen explicit subcycles of the convection terms, resulting in a less accurate coupled solution. The option KIVA2-2 denotes the standard code using the same explicit time step employed in the VNAP2 calculation, a time step that is significantly smaller than the time step calculated internally by KIVA2. As a result, the solution accuracy was greatly improved with only three explicit subcycles of the convection terms required. Finally, the cases KIVA2-3 and KIVA2-4 denote the internally calculated and explicit VNAP2 time steps, respectively, but with a modified version of KIVA2. The code modification consisted of changing the coefficients of the DUAL, DUAF, and DUAB terms in subroutine UFINIT from 0.125 to 0.250 . This modified value was actually in the original version of KIVA2, but the coefficient was changed to 0.125 in later versions of the code.

Based upon the FLUENT results, RAMPANT was only run with a higher-order scheme. The basic GUI path for the higher-order option is given in Table I. In all cases, 
the RAMPANT solutions were solved with second-order spatial discretization of the conservation equations (finite-volume scheme) and an explicit multi-stage Runge-Kutta time integrator.

The VNAP2 code performed the coarse-uniform and coarse-nonuniform mesh solutions without any explicit artificial viscosity. However, the truncation error smoothing inherent to the method was so small on the fine, radially uniform and.fine, radially nonuniform meshes that a small amount of artificial viscosity was required for algorithm stability.

\section{Flow Model, Initial Conditions, and Boundary Conditions}

The fluid-flow model was assumed to be two-dimensional, axisymmetric, and inviscid.

For the time-dependent codes CHAD, KIVA2, and VNAP2, the initial conditions consisted of one-dimensional, isentropic, variable-area flow. The two velocity components were adjusted so that the velocity magnitude remained constant at the onedimensional value and the local flow direction was tangent to the nozzle wall and centerline. Between the wall and centerline, the local flow direction varied linearly. For the time-dependent options with FLUENT and RAMPANT, the initial conditions were obtained from the steady-state options.

For CHAD, KIVA2, and VNAP2, the inlet boundary conditions were the specification of static pressure, density, and flow direction. The one-dimensional values were used for the pressure and density while the inlet flow direction was assumed to be parallel to the centerline. For FLUENT and RAMPANT, the inlet boundary conditions 
were stagnation pressure, stagnation temperature, and inlet flow direction. Because the initial conditions at the exit were supersonic, the various procedures used by the five codes should have little effect on the results. The outer wall was a free-slip boundary condition for velocity and an adiabatic boundary condition for energy transfer.

\section{Computational Mesh}

A stringent test for algorithm accuracy is to compare the code solutions on a number of different meshes. For such a comparison to be meaningful, each code must solve the same problem on the same mesh. Thus, even though CHAD and RAMPANT are unstructured mesh codes, we used a structured, nonuniform mesh with body-fitted coordinates in the radial direction for all the calculations. For the study, VNAP2 was modified to generate a grid file for CHAD, FLUENT and RAMPANT, and KIVA2.

For the comparison among the five codes we investigated four different grids. These four grids are summarized in Table $\Pi$. The first column indicates if the mesh is coarse or fine and radially uniform or radially nonuniform. Column 2 gives the number of axial and radial cells for each mesh. At the throat, the minimum radial zone, which occurs at the nozzle wall, and the maximum radial zone, which occurs at the nozzle centerline, are indicated in columns 3 and 4.

The coarse, radially uniform mesh was 61 axial cells by 10 radial cells. At any point along the axial direction, the radial spacing is uniform along the radial direction. But the uniform radial spacing varies with axial position in one-to-one correspondence with the nozzle wall. Along the axial direction, the axial spacing was adjusted to maintain approximately square cells throughout the entire computational regime. As an example, the coarse, radially uniform mesh is shown in Fig. 1. 
The coarse, radially nonuniform mesh also was 61 axial cells by 10 radial cells with the same axial spacing as the coarse, radially uniform mesh. At each axial position, the radial spacing was stretched to produce a cell at the nozzle wall about one-fourth that of the coarse, radially uniform mesh. Since the number of cells was the same, the radial spacing on the centerline was then about twice the coarse, radially uniform mesh.

The fine, radially uniform mesh was 244 axial cells by 40 radial cells, making the fine-mesh cell size about one-fourth the coarse-mesh cell size. Again, along the axial direction, the axial spacing was adjusted to maintain approximately square cells throughout the computational regime.

The fine, radially nonuniform mesh also was 244 axial cells by 40 radial cells with the same axial spacing as the fine, radially uniform mesh. At each axial position, the radial spacing was stretched to produce a cell at the nozzle wall about one-fourth of the fine, radially uniform mesh. Again, because the number of cells was fixed, this produced radial spacing on the centerline that was about twice that of the fine, radially uniform mesh.

\section{Code-Experiment Error Estimate}

Although it is possible to pick out trends from a visual comparison of the code solutions with the experimental data, it is often quite difficult to determine the most accurate solution. To provide a more definitive statement about solution accuracy, we need some reference to make the comparisons. Since the experimental data are fixed, we will use those data as a common reference. Then, our main comparison among the codes is the Mach number as a function of radius at the nozzle throat. In particular, we calculate the average error for each code as 


$$
\text { average error }(\%)=\frac{100}{N} \sum_{i=1}^{N} \frac{\left|M_{i}^{\text {code }}-M_{i}^{\text {exp }}\right|}{M_{i}^{\exp }},
$$

where $M_{i}^{\text {exp }}$ is the experimental Mach number, $M_{i}^{\text {code }}$ is the code Mach number, and $N$ is the number of experimental points.

One would expect, barring a fundamental implementation or setup error, that the algorithms associated with all five codes would converge to the same solution for a sufficiently fine mesh. Using this metric, all the codes did provide solutions that are quite close on the fine, radially nonuniform mesh, the average difference between the solutions being less than $0.5 \%$.

\section{Comparison Among Codes and Experiment}

For an OCGCNR model, our main interest was code accuracy on a coarse mesh. In three dimensions a fine mesh calculation is not very practical, as the problem becomes too large for scoping and/or prototype design calculations. Furthermore, with a coarse mesh it is possible to calculate the coupled hydrodynamics, radiation transport, and neutron transport on a common grid. As a result, there are more comparison calculations with a coarse mesh than with a fine mesh.

In all cases, it should be understood that the mesh descriptor uniform (nonuniform) means that the mesh is radially uniform (radially nonuniform) with the radial spacing changing in one-to-one correspondence with the nozzle wall. In addition, the axial spacing is adjusted to maintain approximately square cells throughout the computational regime. For all the comparisons, the axial location is at the nozzle throat. In the figures, the solid line represents the most accurate code solution and solid circles 
represent the experimental data. Finally, if we define $\mathbf{r}$ as the radial coordinate at the throat and $\mathrm{R}$ as the throat radius, the dimensionless radius is $\mathrm{r} / \mathrm{R}$.

\section{Coarse Uniform Mesh}

For the coarse uniform mesh, a summary of the Mach number as a function of the dimensionless radius is shown in Fig. 2. Only the most accurate results from FLUENT and KIVA2 are included in Fig 2. The different options for FLUENT and KIVA2 are summarized in Table I. In this case, the solid line represents the most accurate solution obtained from all the codes. The corresponding average errors for all the code options are shown in Fig. 3.

With the coarse uniform mesh, VNAP2 provided the most accurate solution, as seen in both Fig. 2 and Fig. 3. Except at the wall, both the FLUENT and RAMPANT solutions are systematically high relative to the experimental data. Except at the wall, both CHAD and KIVA2 actually provided a more accurate solution than VNAP2. At the wall, however, all the codes except VNAP2 provided a relatively poor solution relative to the interior experimental data points. With a coarse mesh, VNAP2 was expected to provide a better wall solution because the code utilizes a special algorithm at the wall. Because of this superior accuracy at the wall and despite the fact that CHAD and KIVA2 provided a more accurate solution at the interior points, VNAP2 provided the most accurate solution for the entire Mach number profile. In other words, the VNAP2 solution at the interior points was reasonable whereas the solution on the wall was much better than all the other codes.

For the coarse uniform mesh there is significant variation among the different code options, see Fig. 3. As expected, without some care one can obtain a rather poor 
solution on a coarse mesh. Unless a large number of scoping calculations is done, typically one would not compute on such a coarse mesh. However, the level of variation among the code options was somewhat larger than we had expected. For the coarse uniform mesh, VNAP2 provided the most accurate solution, followed by CHAD, KIVA24, FLUENT-2, and RAMPANT.

For the coarse uniform mesh, a summary of the Mach number as a function of the dimensionless radius for the three FLUENT options is shown in Fig. 4. In this figure, the solid line is the most accurate FLUENT solution. All three options of the code provide a relatively poor solution at the wall. The lowest order method appears to be especially poor in the regions where the gradient of the Mach number with respect to radius is large. In this respect, the higher-order interpolation options provide a significant improvement over the lowest-order method.

For the coarse uniform mesh, a summary of the Mach number as a function of the dimensionless radius for the four KIVA2 options is shown in Fig. 5. In this figure, the solid line is the most accurate KIVA2 solution. All four KIVA2 options provide a relatively poor solution on the wall. Surprisingly, the least accurate solution is provided by the standard KIVA2 version, which is the option KIVA2-1 in Table I. The impact of the coefficient change is most significant when the hydrodynamic time-step is internally calculated: compare the pairs KIVA2-1 with KIVA2-3 and KIVA2-2 with KIVA2-4. Basically, the KIVA2-4 option was the only option that gave a reasonable solution. As a result only the KIVA2-4 option was used in the remainder of the study. 


\section{Coarse Nonuniform Mesh}

For the coarse nonuniform mesh, a summary of the Mach number as a function of the dimensionless radius for all the codes is shown in Fig. 6. Only the most accurate solution from FLUENT is shown in Fig. 6. The corresponding errors for all the codes and some of the code options are shown in Fig. 7.

Consistent with the coarse uniform mesh results, Fig. 7 shows that VNAP2 again provided the most accurate solution on the coarse nonuniform mesh. Except at the wall, the FLUENT and RAMPANT solutions remain systematically high relative to the experimental data. For these two codes, the utilization of a nonuniform mesh provided a mixed result at the interior points. The RAMPANT solution is slightly improved but the FLUENT solution is somewhat worse. Furthermore, in contrast to the uniform mesh, where the FLUENT-2 option provided the best overall solution, on the nonuniform mesh the FLUENT-3 option provided the least average error solution. As seen in Fig. 7, however, the difference between these two FLUENT options is insignificant. Except at the wall, CHAD, KIVA2-4, and VNAP2 are now in close agreement with one another and with the experimental data. For these three codes, the utilization of a nonuniform mesh significantly improved the agreement with the interior experimental data, as can be seen by comparing Fig. 2 with Fig. 6 .

Despite these changes at the interior points, the real difference between the uniform and nonuniform meshes occurred at the wall. With the nonuniform mesh, all the code solutions showed a significant improvement with respect to the experimental data at the wall. The RAMPANT solution is actually above the experimental data, whereas all the other solutions remain below the experimental data. Given this trend, the observed 
improvement in the average error is attributed mostly to the improved wall solution, as can be seen by comparing Fig. 2 and Fig. 6.

Despite an improvement in the average error, Fig. 7 indicates that there still remains a significant difference among the solutions on the coarse nonuniform mesh. For the coarse nonuniform mesh, VNAP2 provided the most accurate solution, followed by CHAD, KIVA2-4, FLUENT-3, and RAMPANT. Except for the change from FLUENT-2 to FLUENT-3, this code order is the same as the coarse, uniform mesh.

For the coarse nonuniform mesh, a summary of the Mach number as a function of the dimensionless radius for the three FLUENT options is shown in Fig. 8. At the interior points the FLUENT-2 and FLUENT-3 options are nearly identical. Relative to the coarse uniform mesh, all three FLUENT options provide an improved solution at the wall. Although the solution is improved, the lowest-order method still appears to be especially poor in the regions where the gradient of the Mach number with respect to radius is large. Again, the higher-order interpolation options provide a significant improvement over the lowest-order method.

\section{Fine Uniform Mesh}

The primary motivation for fine mesh calculations was to convince ourselves that all the codes would converge to the same solution. For a fine uniform mesh, the summary of the Mach number as a function of the dimensionless radius is shown in Fig. 9. The solid line represents the most accurate solution obtained from all the code options. Only the most accurate results from FLUENT are included in Fig 9. For this case, the corresponding average errors for all the codes are shown in Fig. 10. 
As a group, all the codes provide solutions that are quite similar on the fine uniform mesh. Consistent with the coarse mesh results, as can be seen from Fig. 10, VNAP2 again provided the most accurate solution. Except at the wall, the RAMPANT solution still appears to be systematically high relative to the experimental data. At the wall, all the solutions remain significantly below the experimental data, including the RAMPANT solution. Although the solutions are similar, as seen in Fig. 10, there remains some slight variation among the different codes and code options. For the fine uniform mesh, VNAP2 provided the most accurate solution, followed by CHAD, KIVA2-4, FLUENT-3, and RAMPANT. Again, this is essentially the same order in accuracy observed for the coarse uniform and nonuniform meshes. So, despite the closer agreement among the codes, the same overall pattern in accuracy still is observed.

For the fine uniform mesh, a summary of the Mach number as a function of the dimensionless radius for the three FLUENT options is shown in Fig. 11. In the figure, the solid line is the most accurate FLUENT solution. Actually, the FLUENT-2 and FLUENT3 options solutions are identical to two significant figures and both appear as the solid line in Fig. 11. For the fine mesh, all three FLUENT options now provide a more consistent set of solutions. In other words, as the mesh is refined, all three options are beginning to approach one another. But, with respect to lowest-order algorithm, the mesh is still not sufficiently refined.

\section{Fine Nonuniform Mesh}

Overall, the solution differences among all the code options were minimized with the fine nonuniform mesh. For this case, a summary of the Mach number as a function of the dimensionless radius is shown in Fig. 12. Only the most accurate results from each 
code are included in Fig 12. The average errors associated with the most accurate solution from each code are shown in Fig. 13.

As with the fine uniform mesh results, as a group all the codes provide solutions that are quite similar. Again, the RAMPANT solution remains systematically high relative to the other solutions. The main difference between the uniform and nonuniform fine mesh solutions occurs at the wall. At the wall the calculated Mach numbers are larger with the nonuniform mesh, which can be seen by comparing Fig. 9 with Fig. 12. This trend is the same as observed on the coarse mesh solutions, although the difference between the uniform and nonuniform mesh becomes less important as the mesh is refined.

With the exception of VNAP2, the average error with the nonuniform mesh is smaller than the average error with the uniform mesh. This can be seen by comparing Fig. 10 and Fig. 13. The nonuniform mesh reduced the average error from 11 to $20 \%$ relative to the fine uniform mesh. Again, this improvement was much less than observed for a coarse mesh, where the improvement varied from 40 to $100 \%$. These results are an indication that the fine, nonuniform mesh solutions are close to being mesh independent. Although the RAMPANT Mach number profile appears to be slightly higher on the average than the other codes, the average error is nearly identical with the other codes. Basically, the RAMPANT solution has less error at the wall whereas all the other code solutions have less error at the interior points.

For the fine nonuniform mesh, CHAD provided the most accurate solution, followed by KIVA2-4, FLUENT-3, RAMPANT, and VNAP2. This is the same order in accuracy as seen on all the meshes, except that VNAP2 went from the best to the worst 
solution. At this point, however, the differences among the average errors have dropped to less than $0.5 \%$.

\section{CHAD - Comparison with Mach Number Contours}

A detailed comparison of the CHAD Mach number solution with the experimental Mach number contours is shown in Fig.14. The solution corresponds to the fine uniform mesh. The computed solution agrees well with the experiment, except for the 1.8, 2.0, and 2.05 Mach number contours. The kink in these experimental contours is due to an oblique shock wave that forms where the circular-arc throat meets the downstream conical section. In the experiment, the tube used to measure the static pressure was actually inclined to the flow near the wall. Although Cuffel et al..$^{5}$ attempted to correct for this inclination, they stated "errors are expected." In addition, the calculations did not resolve the boundary layer, and although such a thin boundary layer usually has little effect on the pressure field, it is unclear if this statement is also true for the weak shock wave/boundary layer interaction. Therefore, the exact nature of this disagreement is unknown.

From an engineering point of view, such an error could impact the local transport of heat into the wall, which would be a critical issue. On the other hand, for a prototypedesign simulation model of the OCGCNR, we know this level of downstream error will not impact the flow upstream of the choked nozzle. ${ }^{4}$

\section{Summary}

For a coarse mesh, VNAP2 and CHAD consistently provided the most accurate solution relative to the experimental data and required no tuning. From a predictive point of view, code accuracy with no tuning on a coarse mesh is an important result. Thus, 
CHAD and VNAP2 can be expected to provide accurate solutions for a convergingdiverging nozzle. On the downside, however, both CHAD and VNAP2 are timedependent codes and are computationally intensive. In this respect, FLUENT and RAMPANT were highly superior in computational efficiency because of a steady-state option. However, the higher-order methods were required to obtain reasonable solution accuracy. With the higher-order methods and a fine, radially nonuniform mesh, both the FLUENT and RAMPANT solutions were as accurate as the CHAD and VNAP2 solutions. Finally, from a predictive point of view, one should be cautious with KIVA2 because of the required algorithm tuning needed to match the experimental data.

The solutions among the codes and code options varied significantly on a coarse mesh. For the meshes considered, a radially nonuniform mesh produced a more accurate solution than a radially uniform mesh with the same number of radial nodes. As would be expected, however, the difference between uniform and nonuniform mesh solutions became less important as the mesh was refined.

On the fine, radially nonuniform mesh, all the codes gave essentially the same average error. Most of the error was associated with the nozzle wall, where the calculated Mach number was consistently lower than the experimental Mach number. Despite the same average error for a fine, radially nonuniform mesh, the calculated Mach number profile across the nozzle throat predicted by RAMPANT remained slightly higher than that calculated by the other codes.

For both fine meshes, all the codes agree well with the Mach number contours measured upstream of the throat. However, none of the codes agrees well with the weak shock located downstream of the throat, although all the codes did indicate the presence 
of a weak shock. The exact nature of this disagreement is unknown. However, none of the calculations resolves the boundary layer. While such a thin boundary layer usually has little effect on the pressure field, it is unclear if this result also is true of the weak shock wave/boundary layer interaction.

\section{Acknowledgments}

This research was jointly funded by the Office of Advanced Space Technology, Marshall Space Flight Center, contract \#H-28025, and the Department of Energy.

\section{References}

1. Thom, K. and Scheider, R. T., Editors, "Proceedings of Symposium on Research on Uranium Plasmas and their Technological Applications," (Gainesville, FL, 1970) NASA SP-236 (1971).

2. Krishnan, M., Editor, "Proceedings of Partially Ionized Plasmas, including the Third Symposium on Uranium Plasmas, " (Princeton University, NJ, September 1976) NASA-CR-157047 (1976).

3. Poston, D. I. and Kamash, T., "A Computational Model for an Open-Cycle Gas Core Nuclear Rocket," Nucl. Sci. Eng. 122, 32 (1996).

4. Thode, L. E., Cline, M. C., and Howe, S. D., "Vortex Formation and Stability in a Scaled Gas-Core Nuclear Rocket Configuration," J. Propulsion and Power 14, 530 (1998).

5. Cuffel, R. F., Back, L. H., and Massier, P. F., "Transonic Flowfield in a Supersonic Nozzle with Small Throat Radius of Curvature," AIAA Journal 7, 1364 (July 1969).

6. Saunders, L. M., "Numerical Solution of the Flow Field in the Throat Region of a Nozzle," BSVD-P-66-TN-001 (NASA CR 82601), Aug. 1966, Brown Engineering Co., Huntsville, AL.

7. Migdal, D., Klein, K., and Moretti, G., "Time-Dependent Calculations for Transonic Nozzle Flow," AIAA Journal 7, 372 (Feb. 1969).

8. Laval, P., "Time-Dependent Calculation Method for Transonic Nozzle Flows," Lecture Notes in Physics 8, 187 (Jan. 1971). 
9. Serra, R. A., "The Determination of Internal Gas Flows by a Transient Numerical Technique," AIAA Journal 10, 603 (May 1972).

10. Cline, M. C., "The Computation of Steady Nozzle Flow by a Time-Dependent Method," AIAA Journal 12, 419 (April 1974).

11. Prozan, R. J. and Kooker, D. E., "The Error Minimization Technique with Application to a Transonic Nozzle Solution," Journal of Fluid Mechanics 43, Pt. 2 , 269 (Aug. 1970).

12. Brown E. F. and Hamilton, G. L., "Survey of Methods for Exhaust-Nozzle Flow Analysis," Journal of Aircraft 13, 4 (Jan. 1976).

13. Sahota, M. S. and ORourke, P. J., "CHAD: Computational Hydrodynamics for Advanced Design," Los Alamos National Laboratory report in preparation.

14. Fluent Incorporated, Centerra Resource Park, 10 Cavendish Court, Lebanon, NH 03766.

15. Amsden, A. A., ORourke, P. J., and Butler, T. D., "KIVA-II: A Computer Program for Chemically Reactive Flows with Sprays," Los Alamos National Laboratory report LA-11560-MS (May 1989).

16. Cline, M. C., "VNAP2: A Computer Program for Computation of TwoDimensional, Time-Dependent, Compressible, Turbulent Flow," Los Alamos National Laboratory report LA-8872 (August 1981). 
Table I. Brief summary of the code options.

\begin{tabular}{|l|l|}
\hline OPTION & DESCRIPTION \\
\hline CHAD & Implicit Eulerian \\
\hline FLUENT - 1 & $\begin{array}{l}\text { Exper/Solution Parameters/Enable Higher Order Schemes/No } \\
\text { Expert/Solution Parameters/Enable Linear Interpolation for Pressure/No }\end{array}$ \\
\hline FLUENT - 2 & $\begin{array}{l}\text { Exper/Solution Parameters/Enable Higher Order Schemes/Yes } \\
\text { Expert/Solution Parameters/Enable Linear Interpolation for Pressure/Yes } \\
\text { Expert/Discretization Schemes/2ND Order Upwind } \\
\text { Expert/Density-Discretization/Density-Interpolation/2nd Order Upwind }\end{array}$ \\
\hline FLUENT - 3 & $\begin{array}{l}\text { Expert/Solution Parameters/Enable Higher Order Schemes/Yes } \\
\text { Expert/Solution Parameters/Enable Linear Interpolation for Pressure/Yes } \\
\text { Expert/Discretization Schemes/Quick } \\
\text { ExpertDensity-Discretization/Density-Interpolation/Quick }\end{array}$ \\
\hline KIVA2-1 & Standard KIVA-II, Coefficient $=0.125$,internal time step \\
\hline KIVA2-2 & Standard KIVA-II, Coefficient $=0.125$, explicit time step from VNAP2 \\
\hline KIVA2-3 & Tuned KIVA-II, Coefficient $=0.250$, internal time step \\
\hline KIVA2-4 & Tuned KIVA-II, Coefficient $=0.250$, explicit time step from VNAP2 \\
\hline RAMPANT & 2nd order accurate spatial discretization - default \\
\hline VNAP2 & coarse mesh - no artificial viscosity ; fine mesh - some artificial viscosity \\
\hline
\end{tabular}


Table II. Summary of the four meshes used in the study.

\begin{tabular}{|l|l|l|l|}
\hline MESH TYPE & CELLS & MIN $(\Delta \mathrm{r})$ & MAX $(\Delta \mathrm{r})$ \\
\hline coarse, radially uniform & $61 \times 10$ & $2.032 \mathrm{e}-3 \mathrm{~cm}$ & $2.032 \mathrm{e}-3 \mathrm{~cm}$ \\
\hline coarse, radially nonuniform & $61 \times 10$ & $5.108 \mathrm{e}-4 \mathrm{~cm}$ & $4.917 \mathrm{e}-3 \mathrm{~cm}$ \\
\hline fine, radially uniform & $244 \times 40$ & $5.080 \mathrm{e}-4 \mathrm{~cm}$ & $5.080 \mathrm{e}-4 \mathrm{~cm}$ \\
\hline fine, radially nonuniform & $244 \times 40$ & $1.270 \mathrm{e}-4 \mathrm{~cm}$ & $1.294 \mathrm{e}-3 \mathrm{~cm}$ \\
\hline
\end{tabular}




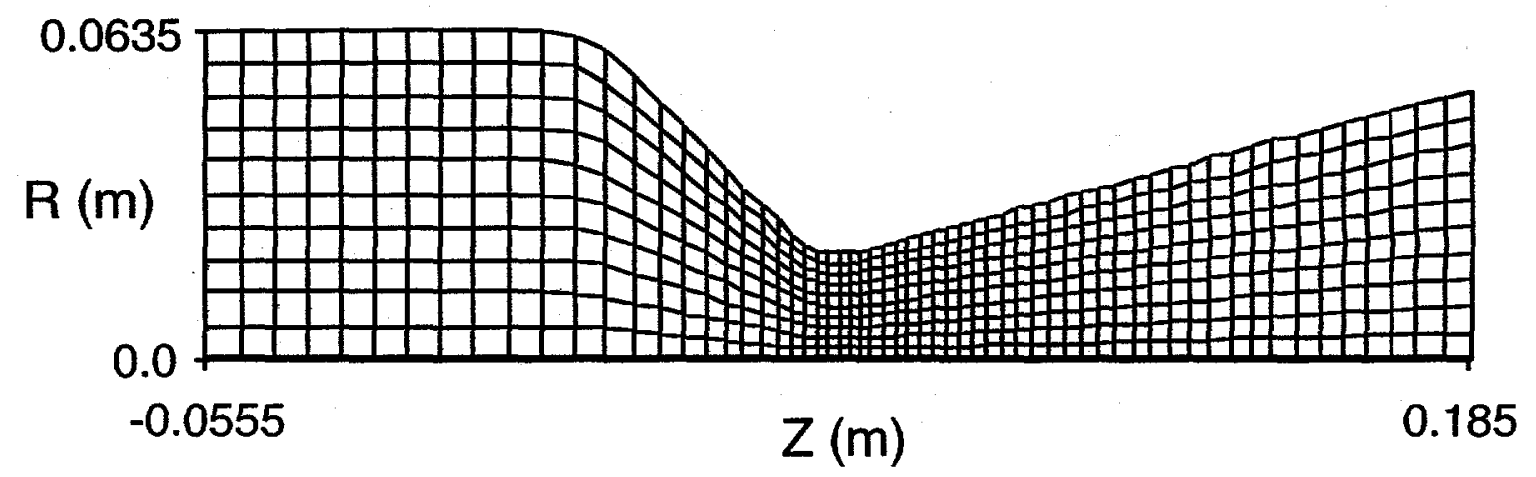

Figure 1. The coarse, radially uniform mesh is shown. At any point along the axial direction, the radial spacing is uniform along the radial direction. 


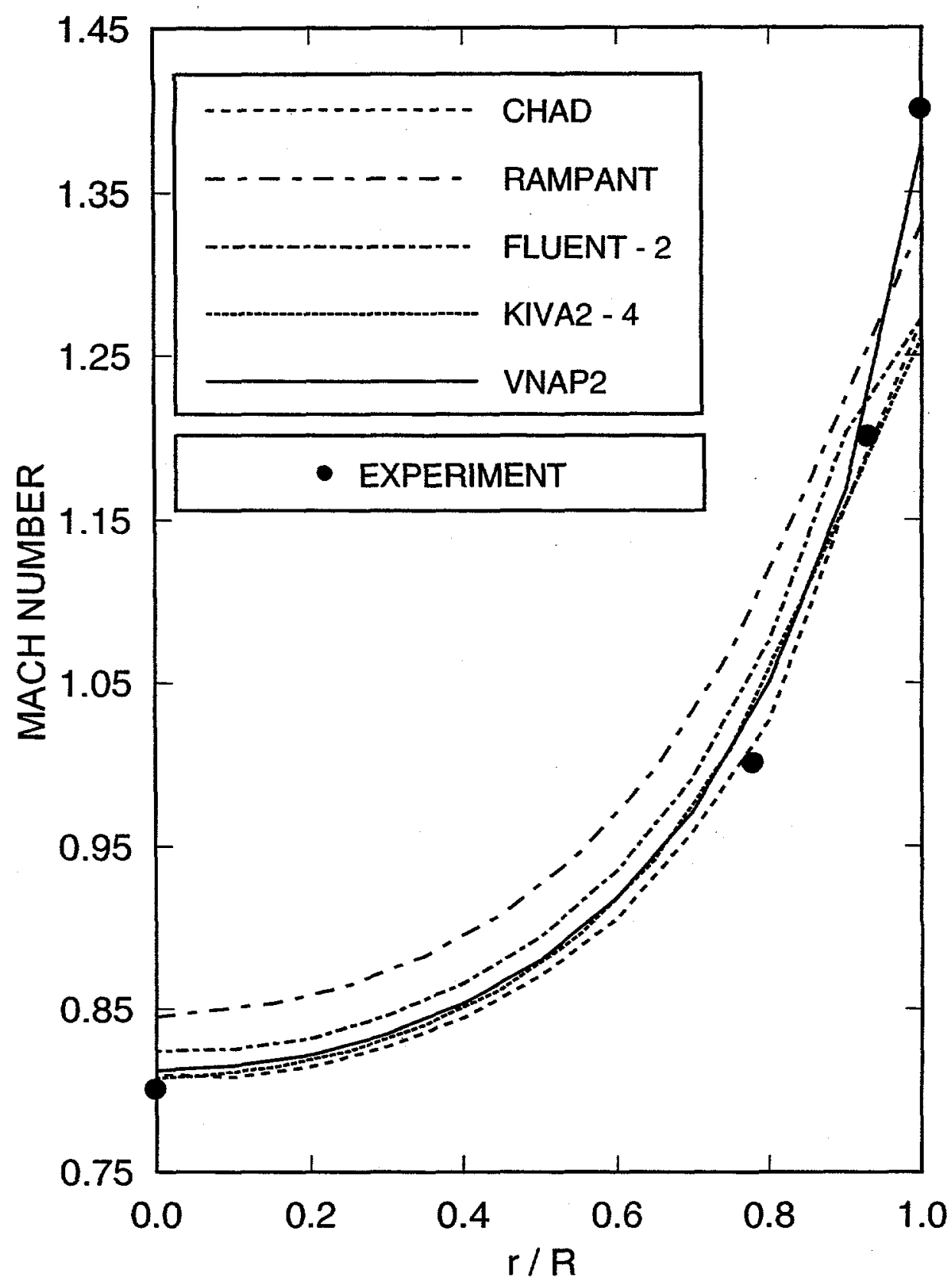

Figure 2. Summary of the Mach number as a function of the dimensionless radius for all the codes on a coarse, radially uniform mesh. The solid line is the most accurate code solution. 


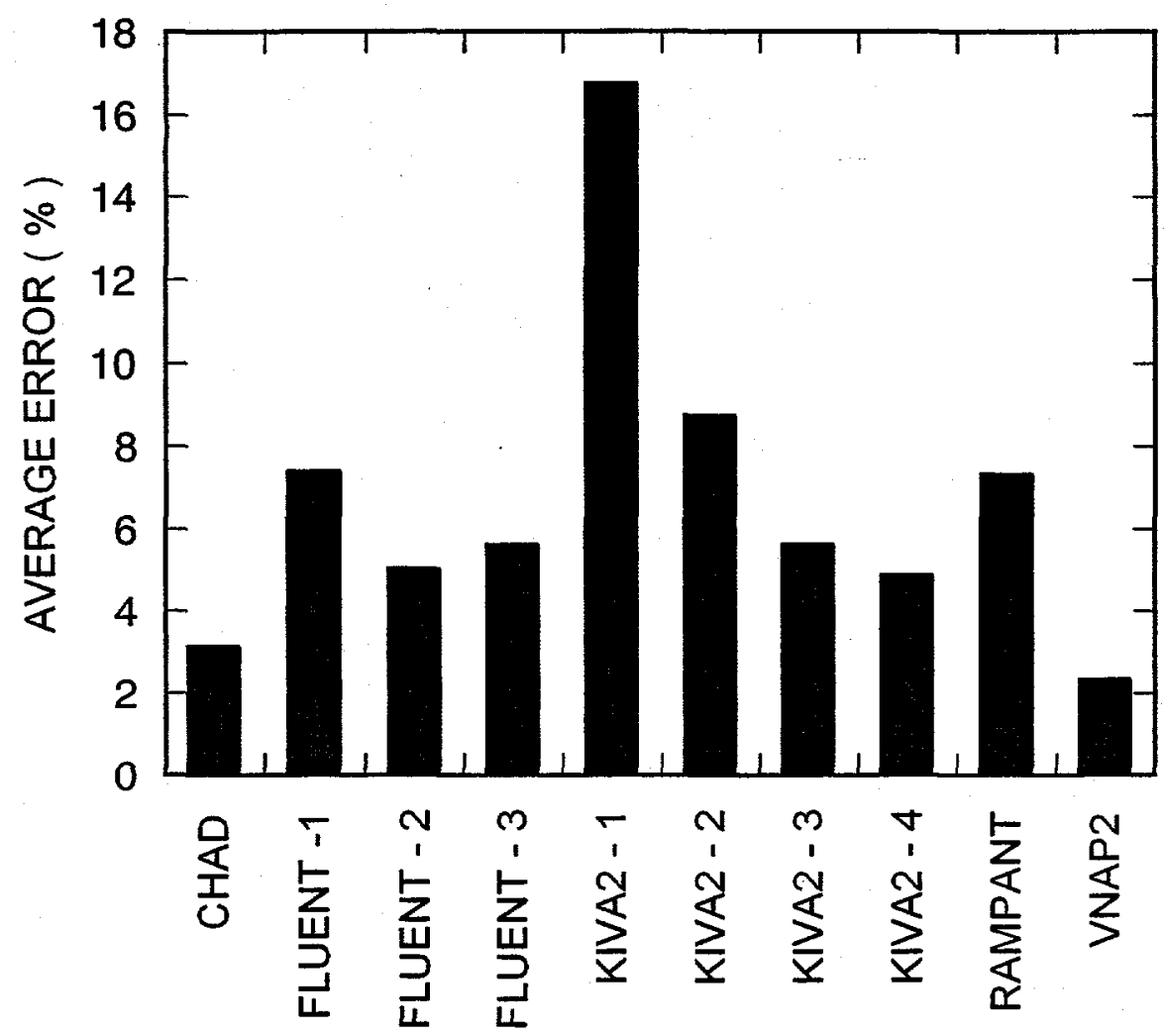

Figure 3. Summary of the average error from the experimental data for all the code options on a coarse, radially uniform mesh. 


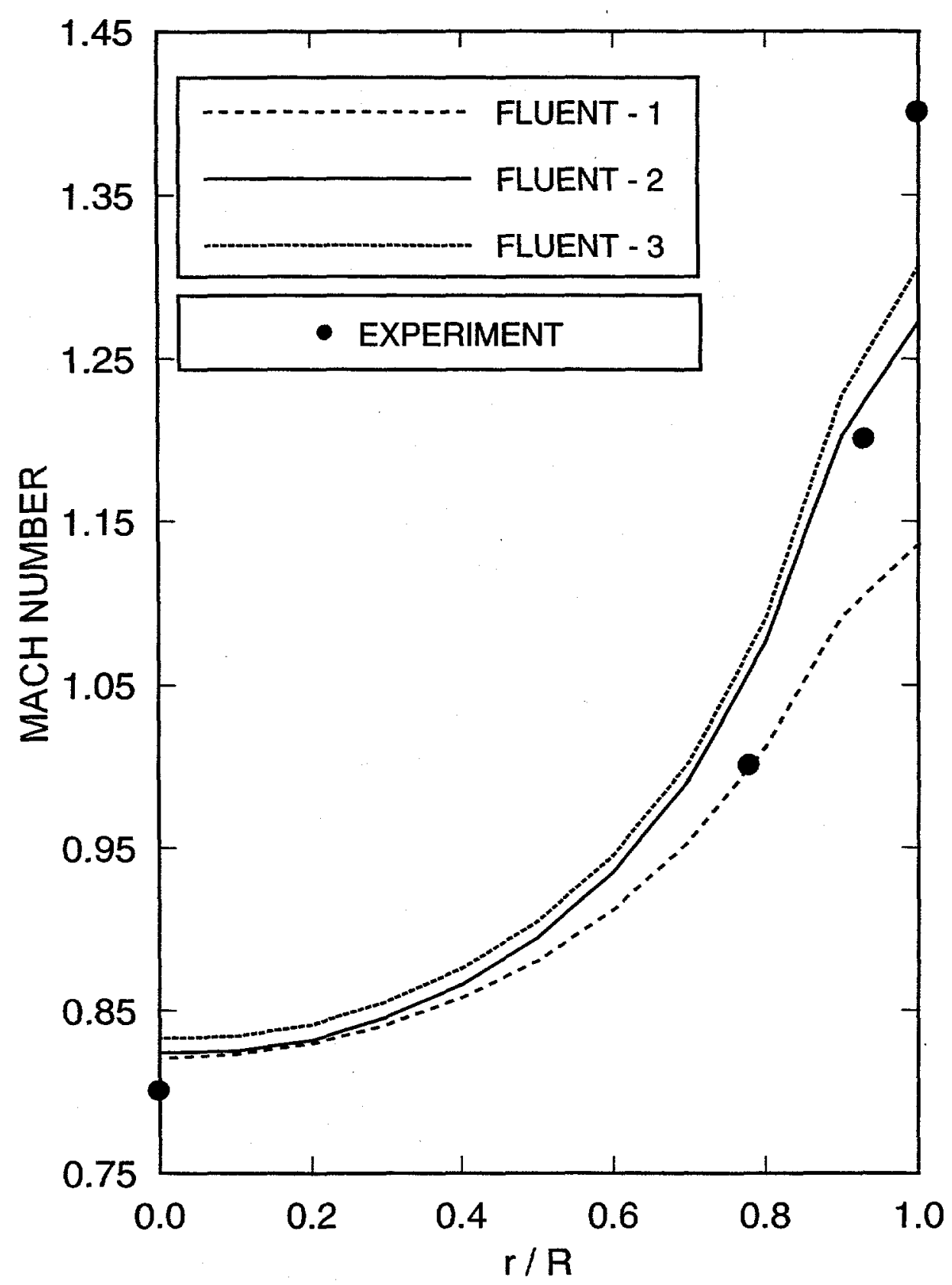

Figure 4. Summary of the Mach number as a function of the dimensionless radius for the three FLUENT options on a coarse, radially uniform mesh. The solid line is the most accurate FLUENT solution. 


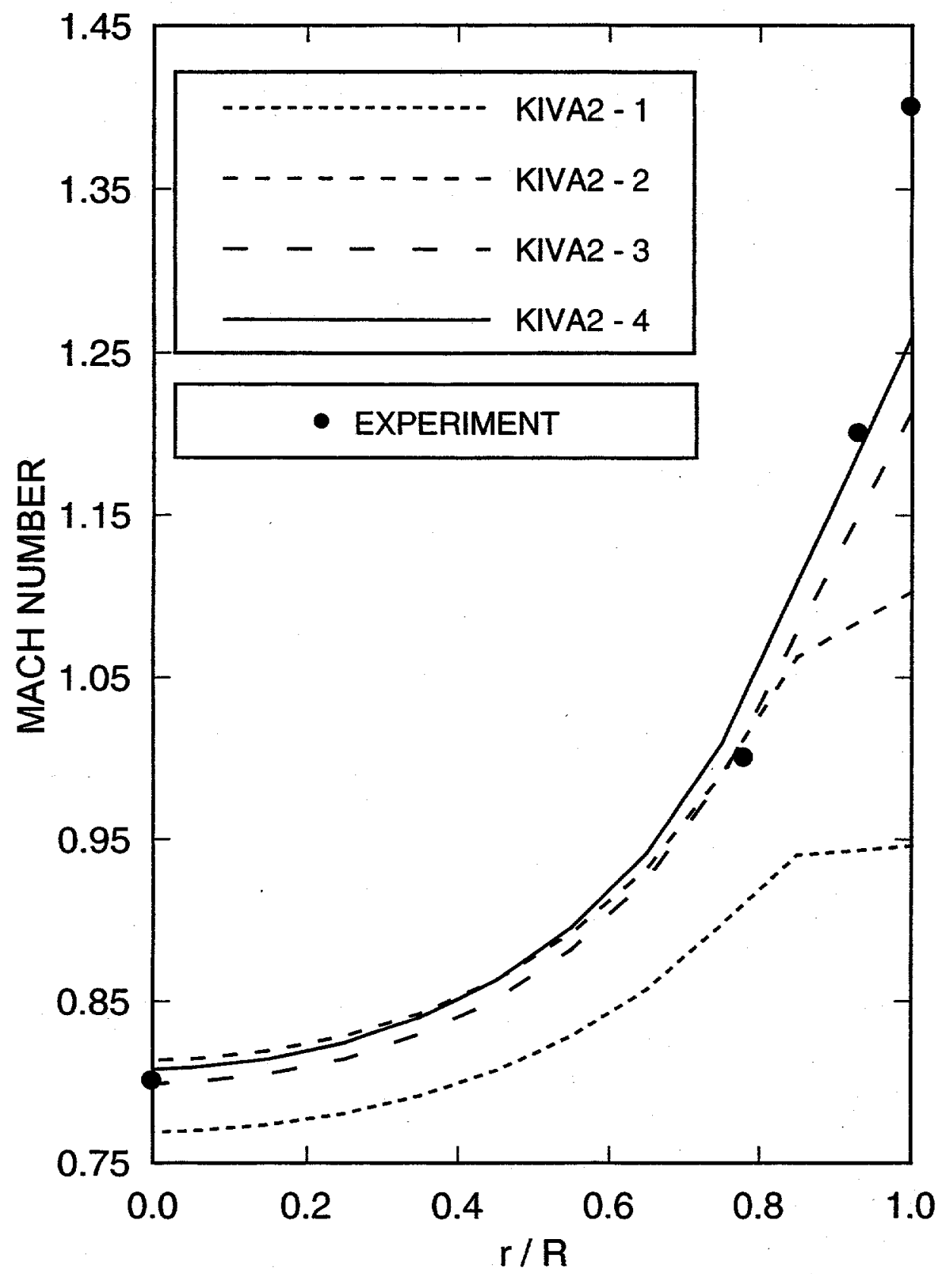

Figure 5. Summary of the Mach number as a function of the dimensionless radius for the four KIVA2 options on a coarse, radially uniform mesh. The solid line is the most accurate KIVA2 solution. 


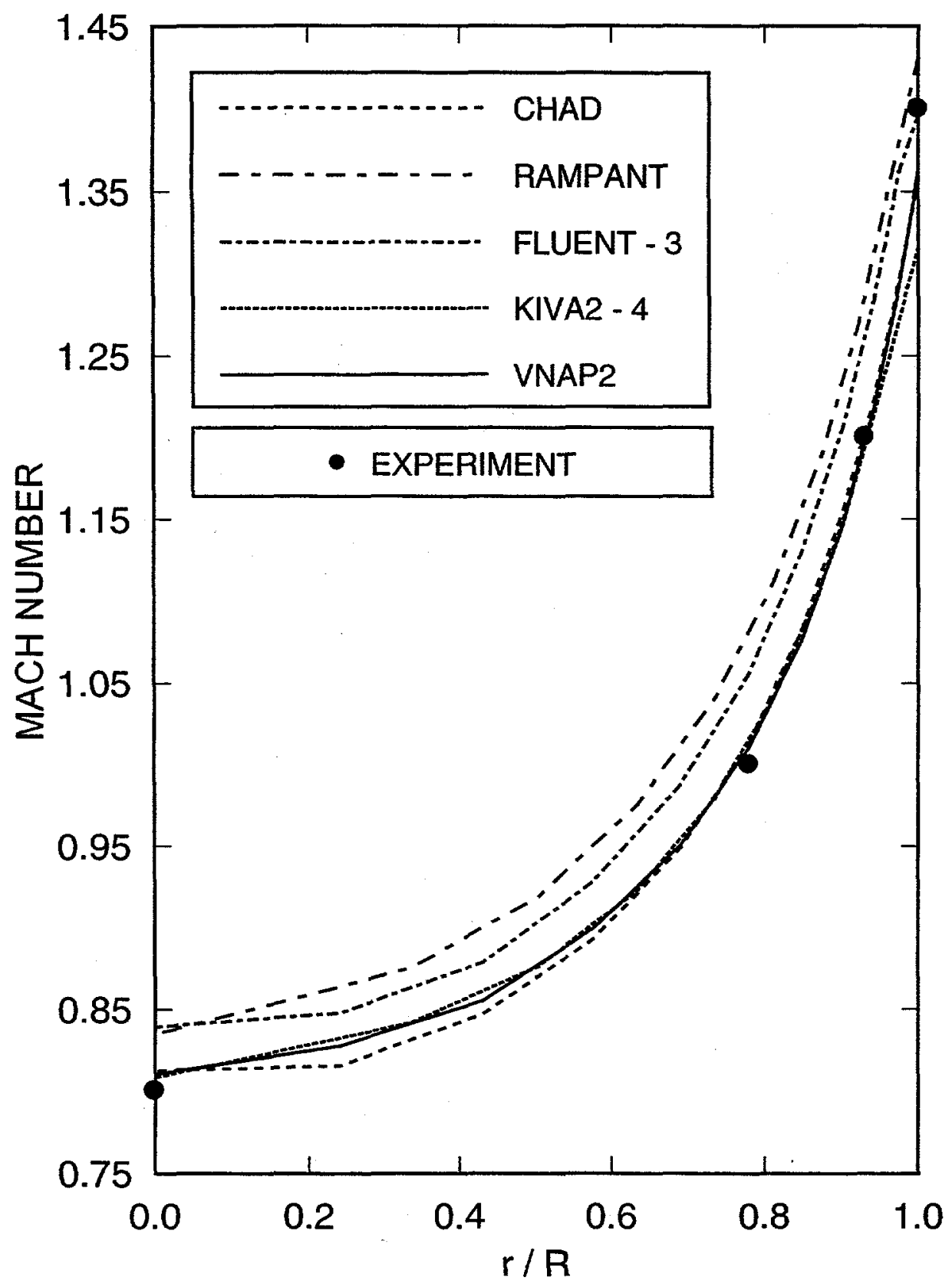

Figure 6. Summary of the Mach number as a function of the dimensionless radius for all the codes on a coarse, radially nonuniform mesh. The solid line is the most accurate code solution. 


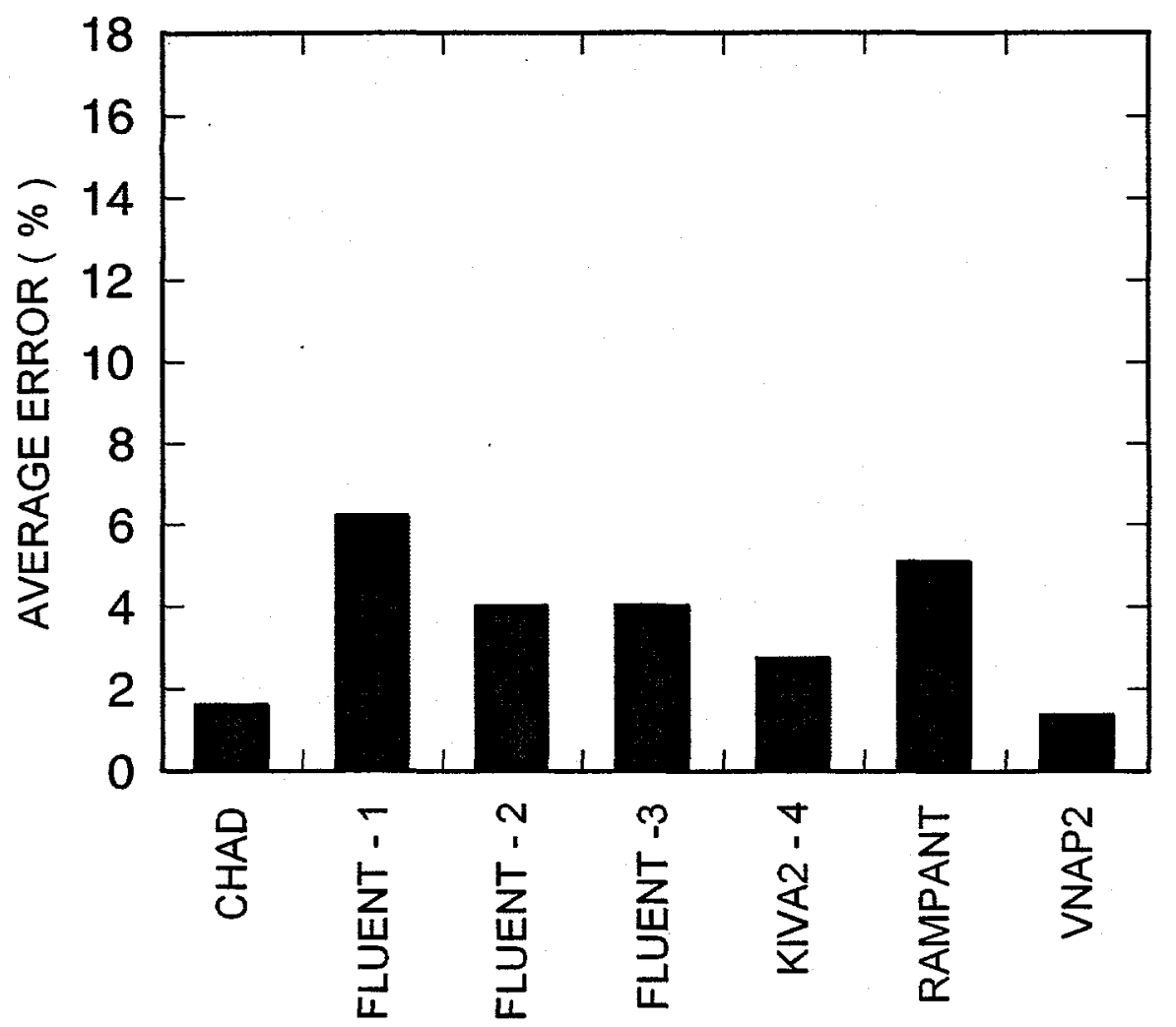

Figure 7. Summary of the average error from the experimental data for different code options on a coarse, radially nonuniform mesh. 


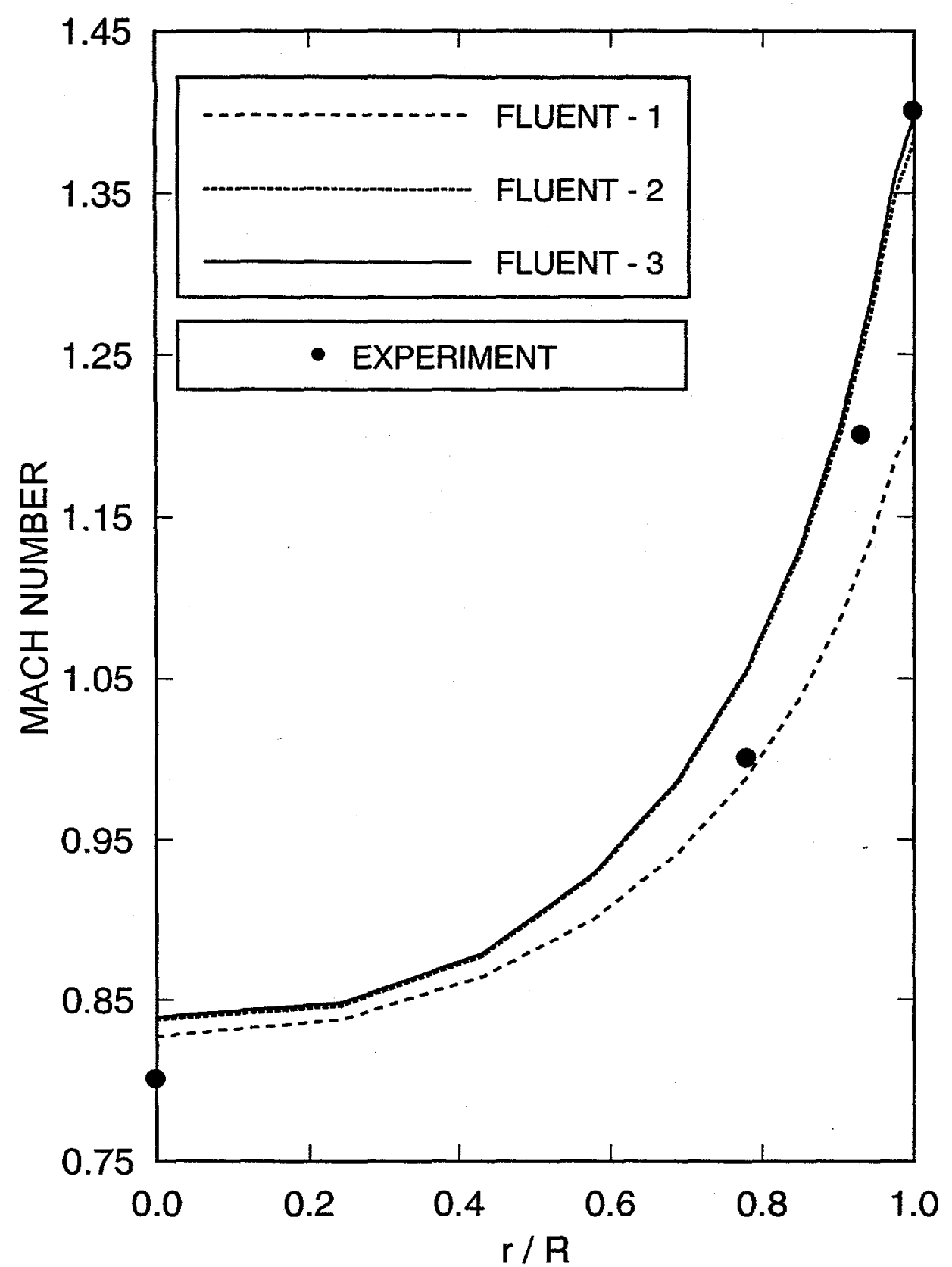

Figure 8. Summary of the Mach number as a function of the dimensionless radius for the three FLUENT options on a coarse, radially nonuniform mesh. The solid line is the most accurate FLUENT solution. 


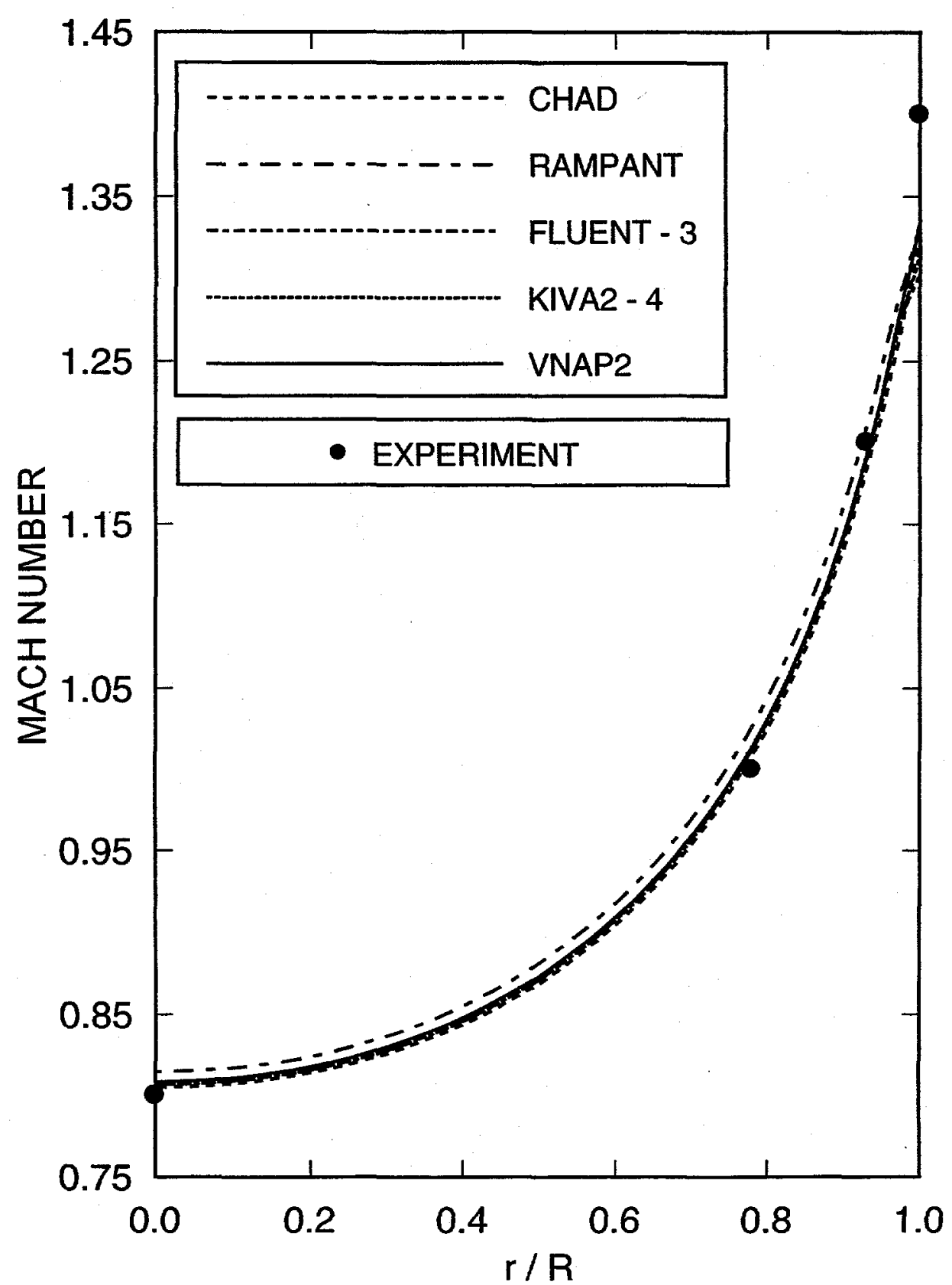

Figure.9. Summary of the Mach number as a function of the dimensionless radius for all the codes on a fine, radially uniform mesh. The solid line is the most accurate code solution. 


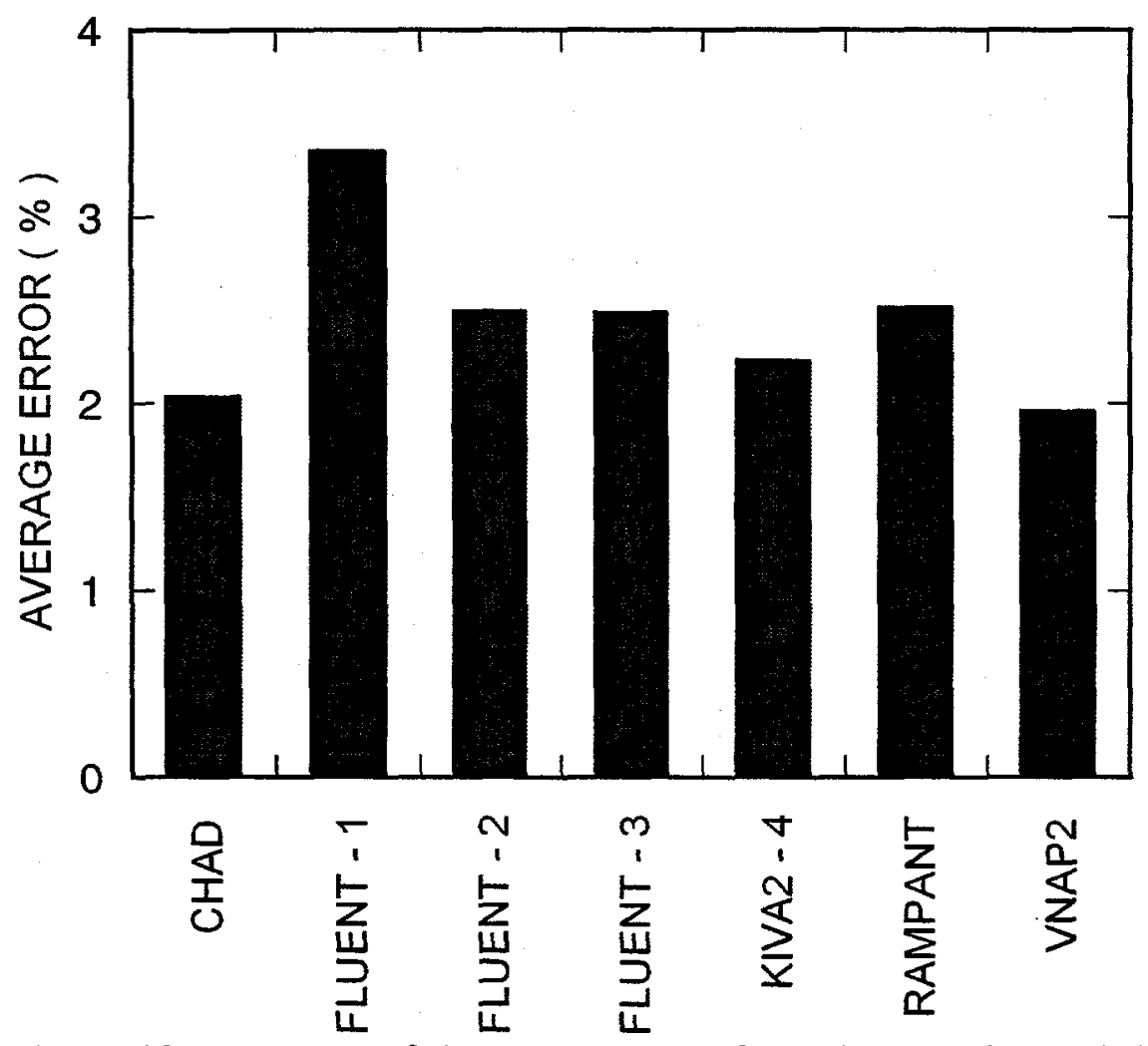

Figure 10. Summary of the average error from the experimental data for different code options on a fine, radially uniform mesh. 


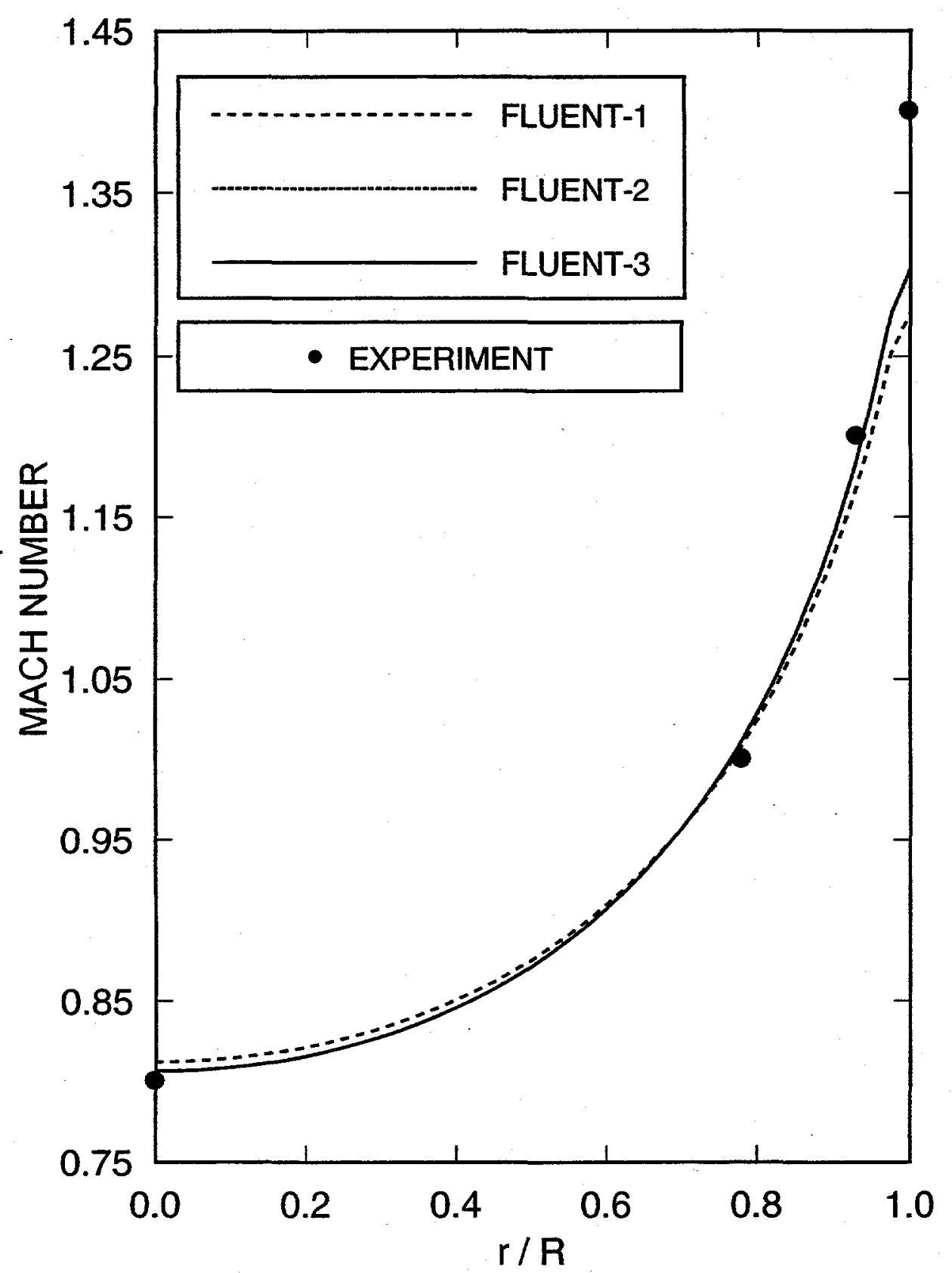

Figure 11. Summary of the Mach number as a function of the dimensionless radius for the three FLUENT options on a fine, radially uniform mesh. The solid line is the most accurate FLUENT solution. 


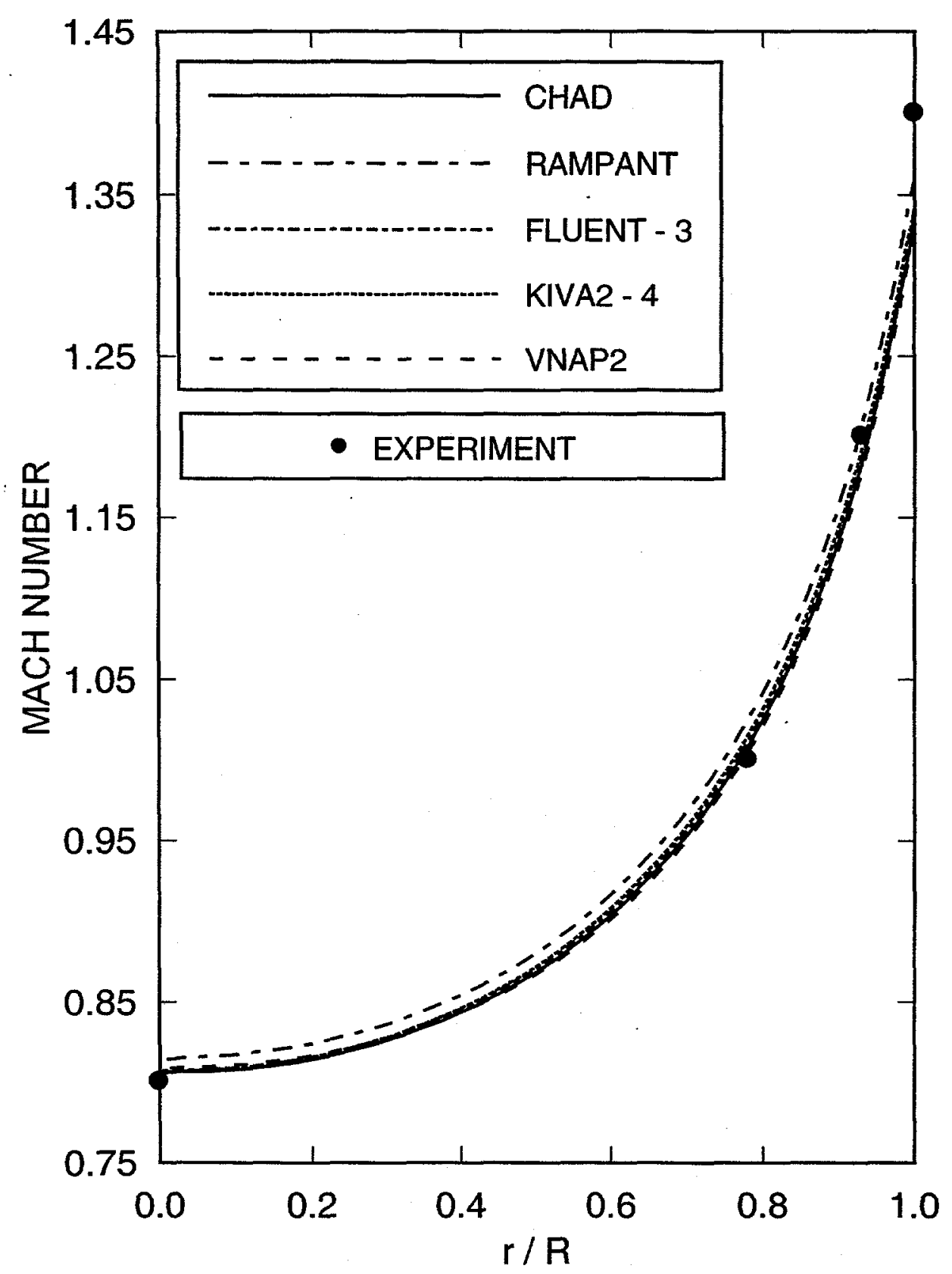

Figure 12. Summary of the Mach number as a function of the dimensionless radius for all the codes on a fine, radially nonuniform mesh. The solid line is the most accurate code solution. 


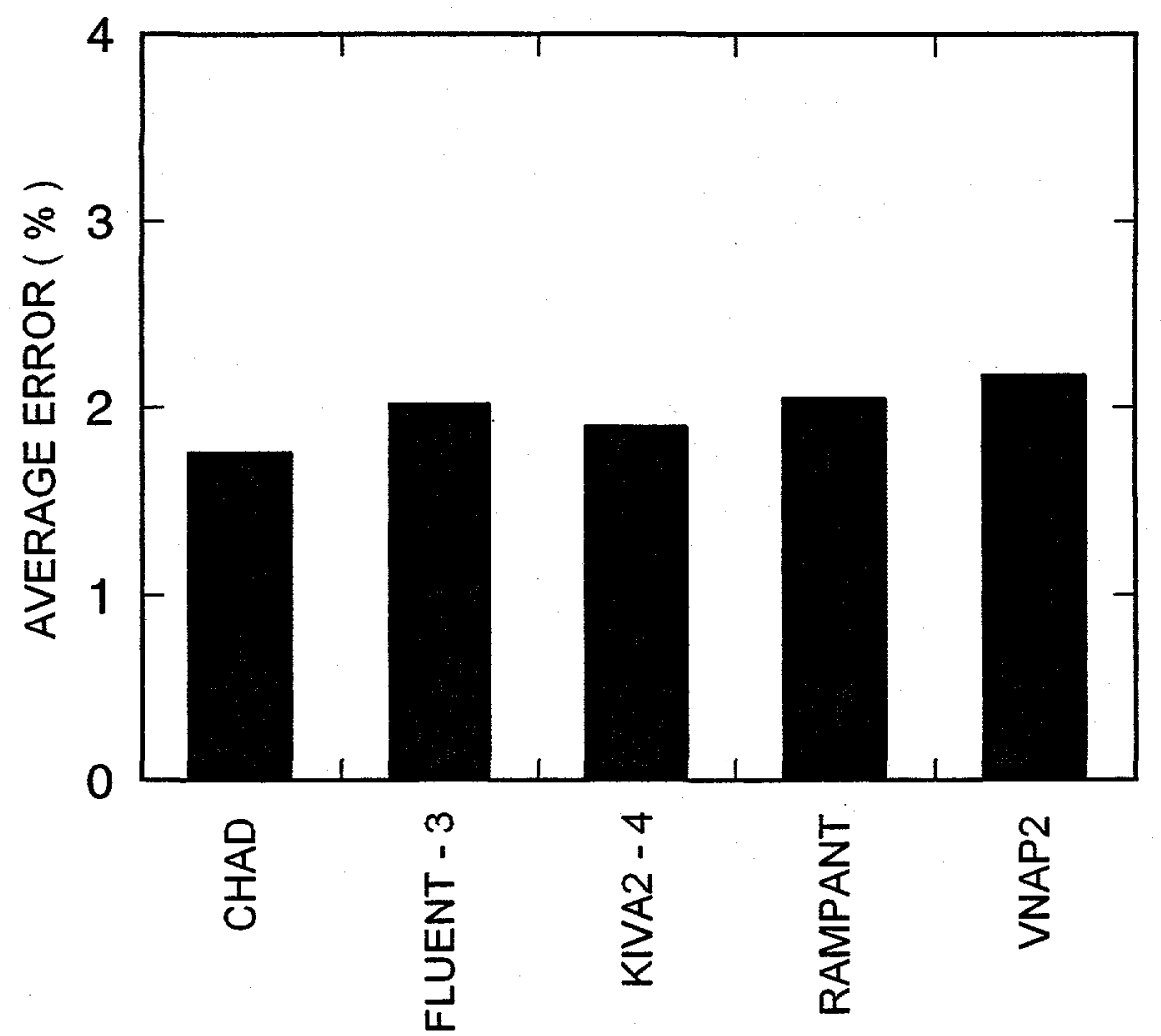

Figure 13. Summary of the average error from the experimental data for different code options on a fine, radially nonuniform mesh. 


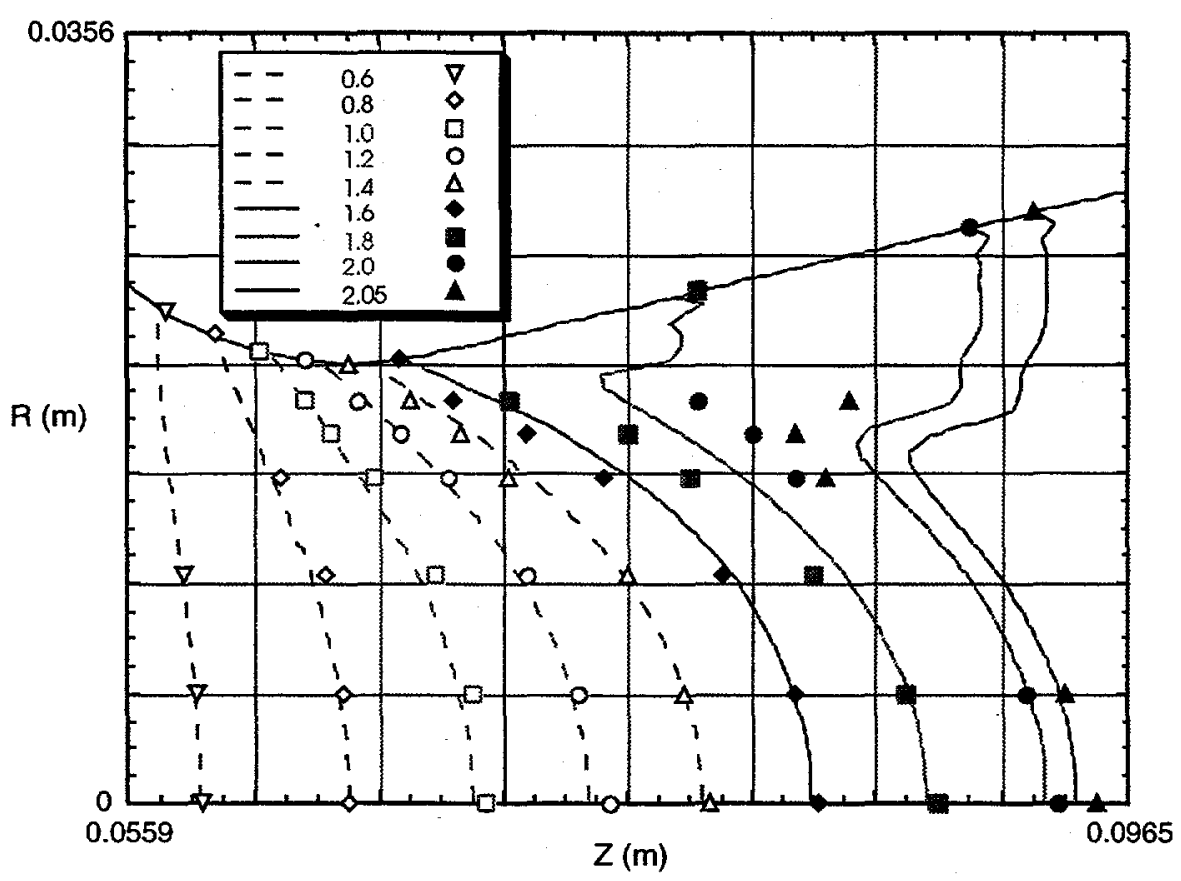

Figure 14. Comparison of experimental data points (symbols) and CHAD-calculated Mach number contours on a fine, radially uniform mesh. 